\title{
A Comparative Study of User Experience According to One- person Media Virtual YouTuber (VTuber) and General YouTuber
}

\author{
Dong-Yoon Kim ${ }^{1}$, Hoon-Sik Yoo ${ }^{2}$ \\ ${ }^{1}$ Student, Department of New Media, Seoul Media Institute of Technology(SMIT), South of Korea, \\ dongyoon0830@gmail.com \\ ${ }^{2}$ Professor, Department of New Media, Seoul Media Institute of Technology(SMIT), South of Korea, \\ hsyoo@smit.ac.kr
}

Corresponding author: Hoon-Sik Yoo

\begin{abstract}
With the spread of advanced computer graphics (CG) technology and online video platforms' popularization, 3D content is emerging in the one-person broadcast field. A new type of content area called Virtual YouTuber (VTuber) using VR equipment has emerged. Therefore, this study is aimed to analyze the differences in content user experience quantitatively and qualitatively by comparing existing general and virtual YouTubers. A survey method was employed to collect data using a questionnaire developed by the AttrakDiff evaluation method. The questionnaire was designed to measure four dimensions of the usability (pragmatic quality, hedonic quality-identity, hedonic quality-simulation, and attractiveness) with 28 items (pairs of opposite adjectives positioned at both ends) rated on a 7-point scale (-3: negative adjective $\sim 3$ : positive adjective). A total of 50 people ( 25 men and 25 women) in their 20 s to 30 s participated in the survey. The results of the study revealed the characteristics of the user experience of ordinary and virtual YouTubers. This work is significant as an attempt to further identify the preferences and features of the user experience using AttrakDiff evaluation techniques from the simple rating comparison for existing virtual YouTuber (Vtuber). Starting with this research, we hope that more related research will be conducted on virtual YouTuber (VTuber).
\end{abstract}

Keywords: One-person Broadcast, Virtual YouTuber (VTuber), AttrakDiff, Usability Evaluation, $\mathrm{UX} / \mathrm{UI}$

\section{Introduction}

Today, the general public's hurdles to access CG technology have decreased with the rapid development and spread of the technology for processing computer graphics. Anyone can easily distribute one-person broadcast and video content since online video platforms such as Africa TV and YouTube have developed. As advanced computer graphics performance, inexpensive devices, and video service platforms' development create synergy with each other, a myriad of 3D contents and services expressed in CG is being created. A new type of video content area called Virtual YouTuber (VTuber) using VR equipment is emerging[1].

As a result, research on virtual YouTubers (VTuber) using virtual avatars is being conducted. Among them, there was a prior study to compare the differences in people's responses when a virtual YouTuber

Received: December 14, 2020; $1^{\text {st }}$ Review Result: January 27, 2021; $2^{\text {nd }}$ Review Result: March 18, 2021 Accepted: April 30, 2021 
(VTuber) and a regular YouTuber, but it was regrettable that the results were derived by simply comparing and analyzing the ratings. This study seeks to explore how people's response differences differ in quantitative and qualitative evaluation methods when conducted by a virtual YouTuber (VTuber) and a regular YouTuber using the AttrakDiff evaluation method. The questions in this study are as follows.

1. Vtuber via virtual avatar is closer to the viewer's preference, positive or negative words, compared to conventional YouTubers?

2. Which of the qualitative factors (practicality, identity, irritability, aesthetics) does Vtuber through virtual avatar show when compared to conventional YouTubers?

\section{Theoretical Background}

\subsection{VR Technology and One-person Media Research}

The commercialization of $5 \mathrm{G}$ services that have improved mobile communication technology's speed and data capacity has made it possible to process more extensive data. Accordingly, the content industry related to virtual reality (VR) technology has been activated, and production is expanding from the military, medical, and construction fields to new fields such as games, movies, and sports. Although the hurdles accessing CG technology are said to have decreased, it is inevitable for users in the case of VR technology to wear head-mounted display (HMD), resulting in various inconveniences. Furthermore, with the running cost of unfamiliar media technology being present, the VR industry is still experiencing difficulties growing and being popularized[2].

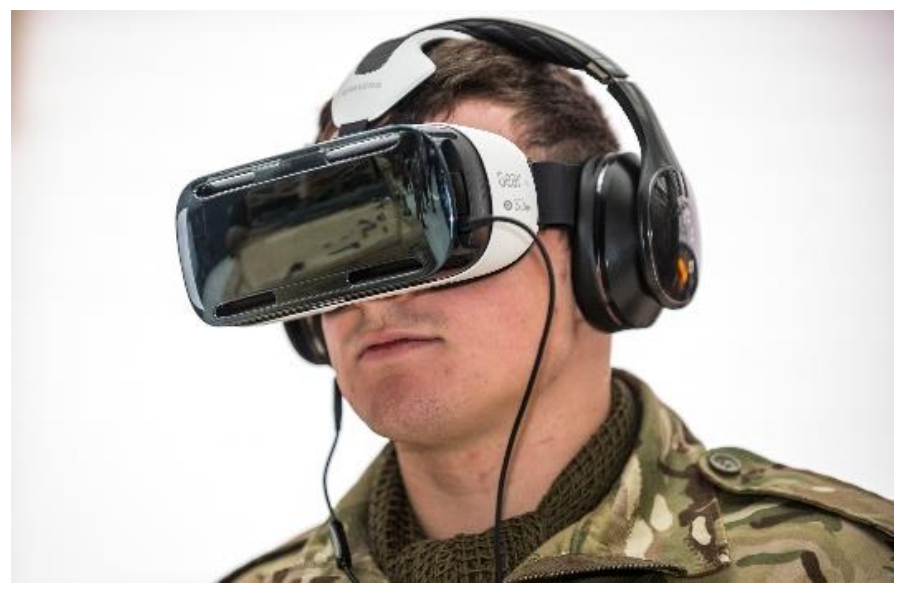

[Fig. 1] Picture of Wearing an HMD Using VR

Nevertheless, the Millennials and Gen Z, who are now called the MZ generation, handle smartphones well and are used to communicating with video as they are a generation who watches YouTube. Notably, they are a generation that can actively accept one-person media using VR technology because they are digital natives born and grown in a digital environment and are open to and actively accepting new and unfamiliar media technologies[2].

\subsection{Definition of Virtual YouTuber (VTuber)}

Virtual Youtuber refers to a virtual avatar who forms a virtual character and conducts internet broadcasting, using Motion Capture technology and CG technology. This technology recognizes an 
object's motion information by attaching a sensor to the object and reproduces it in images mainly used in movies, animations, and games. The term VTuber was first used by Kizuna Ai in Japan in 2016 and is called VTuber by shortening the virtual YouTuber. They communicate with people through internet broadcasting channels[3].

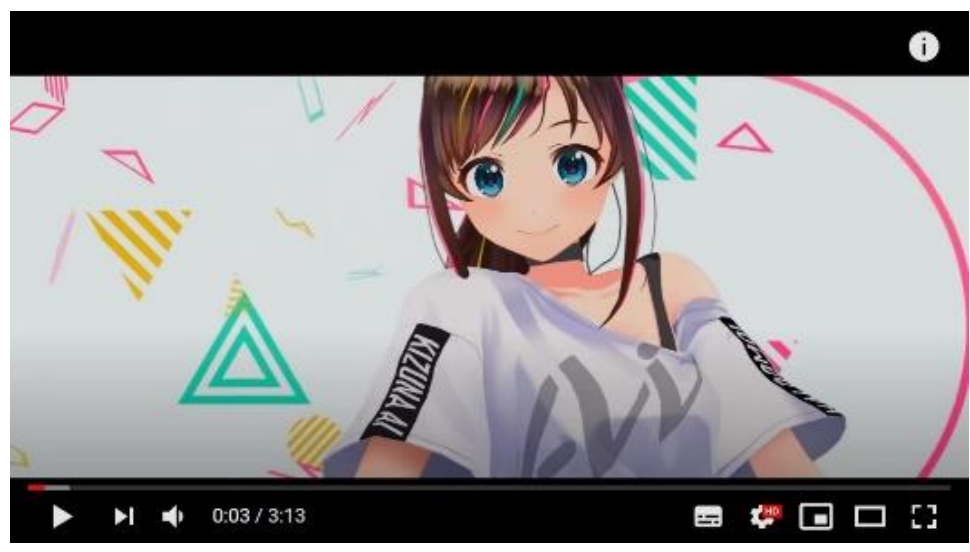

[Fig. 2] Japanese Virtual YouTuber (VTuber) Kizuna Ai

\subsection{The Case of Virtual YouTuber (VTuber)}

Virtual YouTuber broadcasts on the Internet on various topics; a virtual character (avatar) expressed in 3D rather than a real person becomes a streamer through motion capture technology using VR equipment. VTuber contents are increasing. Currently, VTuber are active as a global content creator in several countries, including Japan, Korea, and the United States, and mainly generates revenues through advertising[4].

Representative virtual YouTubers in Japan include Kizuna Ai, Luna Kaguya, Akari Mirai, Shiro, and Nekomiya Hinata. Representative virtual YouTubers in Korea include SeAH and Shuble. Virtual YouTuber is particularly significant in that it has discovered a new content market as it is being used in $\mathrm{TV}$, product development/production, music broadcasting, and mobile games. For this reason, several companies are trying to enter the new video content market by developing a Virtual YouTuber (VTuber)[4].

Among the Virtual YouTuber cases in South Korea, the game 'Elsword 'showed the potential as a star character of VTuber by providing music and video services via personal Internet broadcasting, YouTube, and affiliates through the Elstar project; the use of games also increased. Besides, SeAH's YouTube channel $<$ SeAH Story $>$ recorded 68,500 subscribers as of October 4, 2020, and Schubl's YouTube channel Schubl recorded 74,200 subscribers as of October 04, 2020. Virtual YouTuber content using star characters like these is gaining enthusiastic responses from the MZ generation consumers. It is because the copyright in character, intellectual property rights in South Korea works familiar to the MZ generation that currently consumes VTuber contents, and it seems that it has the potential to expand as VR video content using the character star system business model[2].

\section{Methods}

\subsection{Survey}

The purpose of this study is to compare virtual YouTuber (VTuber) and regular YouTubers to verify differences in people's responses with quantitative and qualitative evaluation methods. Therefore, the 
subjects of this study are persons with no experience in virtual YouTuber (VTuber) content, and the sample was selected as a target for Koreans who have experience in watching social media videos. From September 23 to 27, 2020, a total of 52 persons agreed to participate in the study and answered a direct survey using the online Google questionnaire. The method of recruiting the subjects was written by explaining the purpose of the research to the general public and distributing structured online Google questionnaires to those who voluntarily wish to participate. A total of 50 copies (96.2\%), excluding two of the unfaithful responses, were selected for final analysis out of the 52 completed questionnaires.

A look at the general features of 50 survey respondents included in the final analysis of this study is as follows.

The subjects of the study are a total of 50 ordinary Koreans who have watched social media videos but have no experience in virtual YouTuber content, with 25 men and women (50.0\%) each. In the age group, people in their $20 \mathrm{~s}$ accounted for the largest portion of the total respondents with $64.0 \%$, followed by those in their $30 \mathrm{~s}$ with $36.0 \%$.

The demographic information of the subjects studied as shown above is summarized in [Table 1].

[Table 1] Demographic Information of the Participants

\begin{tabular}{|c|c|c|c|}
\hline \multicolumn{2}{|c|}{ Group } & Frequency & Percentage (\%) \\
\hline \multirow{3}{*}{ Sex } & Male & 25 & 50 \\
\cline { 2 - 4 } & Female & 25 & 50 \\
\cline { 2 - 4 } & Total & 50 & 100 \\
\hline \multirow{3}{*}{ Age } & $20 \mathrm{~s}$ & 32 & 64 \\
\cline { 2 - 4 } & $30 \mathrm{~s}$ & 18 & 36 \\
\cline { 2 - 4 } & Total & 50 & 100 \\
\hline
\end{tabular}

\subsection{Measurement Tools and Method}

Ease of use, or optimal manipulation, is an integral part of interactive products, and more and more products require user experience. Nowadays, it is necessary to apply an evaluation method that records both recognized practical quality, pleasant quality, and interactive products. The AttrakDiff evaluation methodology supports three scenarios before and after a single evaluation, product comparison, and comparison, helping users understand the usability and design of interactive products. Therefore, oneperson broadcasting was classified as an interactive family that produces content that viewers are interested in and like as a two-way communication with viewers, and therefore, it was most appropriate to use AttrakDiff evaluation methodology in this study to compare user experiences between virtual YouTubers and ordinary YouTubers).

\subsection{Virtual YouTuber and General YouTuber Videos}

Video of a virtual YouTuber (VTuber) and a regular YouTuber were prepared, and each video was 1 minute long, with four videos uploaded to YouTube as a single video, and the subjects were asked to watch the link before conducting the experiment. Video's virtual YouTuber (VTuber) selected four channels with the highest average ratings among one-person broadcasts, including using virtual avatars. 
The criteria for selecting a virtual YouTuber (VTuber) channel using each virtual avatar consisted of the top $1 \%$ of YouTubers in the field with more than 50,000 subscribers. Similarly, the video's general YouTuber selected four contents of each genre with the highest average ratings among one-person broadcasts, including games, eating shows, vlogs, and beauty. The criteria for selecting general YouTuber cases that produce content for each genre consisted of YouTubers belonging to the top 1\% of the top $1 \%$ in the field with more than 1 million subscribers.

\subsection{Develop Questionnaire Questions}

The composition of the questionnaires used in this examination is as follows: The AttrakDiff usability evaluation method consisted of seven questions each in four categories: practicality, identity, irritability, and aesthetic. The total questions were 35 questions, consisting of a seven-point Likert scale (-3: negative words to 3 : positive words), and allowed them to choose the degree to which they thought they were applicable in a self-written manner. AttrakDiff provides a usability assessment tool for interactive products and services and evaluates 28 pairs of items divided into four categories: PQ (pragmatic quality), HQ-I (hedonic quality-identity), HQ-S (hedonic quality-simulation), and ATT (attractivity).

The definitions of the four components in [Table 2] are as follows.

1) Pragmatic Quality (pragmatic quality): It is about practicality, how useful and easy to use, and whether it can be managed.

2) Hedonic Quality-Identity (hedonic quality-identity): An evaluation of how helpful it is to establish relationships with other people.

3) Hedonic Quality-Stimulation (hedonic quality-simulation): An evaluation of how much you stimulate yourself in doing something.

4) Attractiveness (attractiveness): An evaluation of attractiveness, such as how attractive and pretty you think the product is.

[Table 2] Measurement Items for the AttrakDiff Usability Evaluation

\begin{tabular}{|c|c|c|c|c|}
\hline & Pragmatic quality & Hedonic quality-identity & $\begin{array}{l}\text { Hedonic quality- } \\
\text { simulation }\end{array}$ & Attractiveness \\
\hline \multirow{7}{*}{ Items } & Technical - human & Isolating - connective & Conventional - inventive & Unpleasant - pleasant \\
\hline & Complicated - simple & $\begin{array}{l}\text { Unprofessional - } \\
\text { professional }\end{array}$ & Unimaginative - creative & Ugly - attractive \\
\hline & Impractical-practical & Tacky - stylish & Cautious - bold & Disagreeable - likeable \\
\hline & $\begin{array}{l}\text { Cumbersome - } \\
\text { straightforward }\end{array}$ & Cheap - premium & Conservative - innovative & Rejecting - inviting \\
\hline & $\begin{array}{l}\text { Unpredictable - } \\
\text { predictable }\end{array}$ & Alienating - integrating & Dull - captivating & Bad-good \\
\hline & $\begin{array}{l}\text { Confusing - clearly } \\
\text { structured }\end{array}$ & $\begin{array}{l}\text { Separates me from people } \\
\text { - brings me closer to } \\
\text { people }\end{array}$ & $\begin{array}{l}\text { Undemanding - } \\
\text { challenging }\end{array}$ & Repelling - appealing \\
\hline & Unruly - manageable & $\begin{array}{l}\text { Unpresentable - } \\
\text { presentable }\end{array}$ & Ordinary-novel & Discouraging - motivating \\
\hline
\end{tabular}




\section{Results}

\subsection{AttrakDiff Evaluation Results}

\subsubsection{Evaluation Result by Item}

As a result of analyzing the results based on the 7-point scale (-3: negative word $\sim 3$ : positive word at both ends), the study obtained the results on pragmatic quality, hedonic quality-simulation, hedonic quality-identity, and attractiveness for each of the general YouTuber and the Virtual YouTuber (VTuber), as presented in [Table 3] and [Fig. 3].

[Table 3] AttrakDiff Evaluation Results of General YouTuber and Virtual YouTuber (VTuber)

\begin{tabular}{|c|c|c|c|c|}
\hline Contents & $\begin{array}{c}\text { Pragmatic quality } \\
\text { PQ }\end{array}$ & $\begin{array}{c}\text { Hedonic quality- } \\
\text { identity } \\
\text { HQ-I }\end{array}$ & $\begin{array}{c}\text { Hedonic quality- } \\
\text { simulation } \\
\text { HQ-S }\end{array}$ & $\begin{array}{c}\text { Attractiveness } \\
\text { ATT }\end{array}$ \\
\hline Virtual YouTuber (VTuber) & 0.15 & 0.81 & 0.25 & 0.73 \\
\hline General YouTuber & 1.51 & 1.11 & 1.55 & 1.65 \\
\hline
\end{tabular}

\section{Diagram of average values}

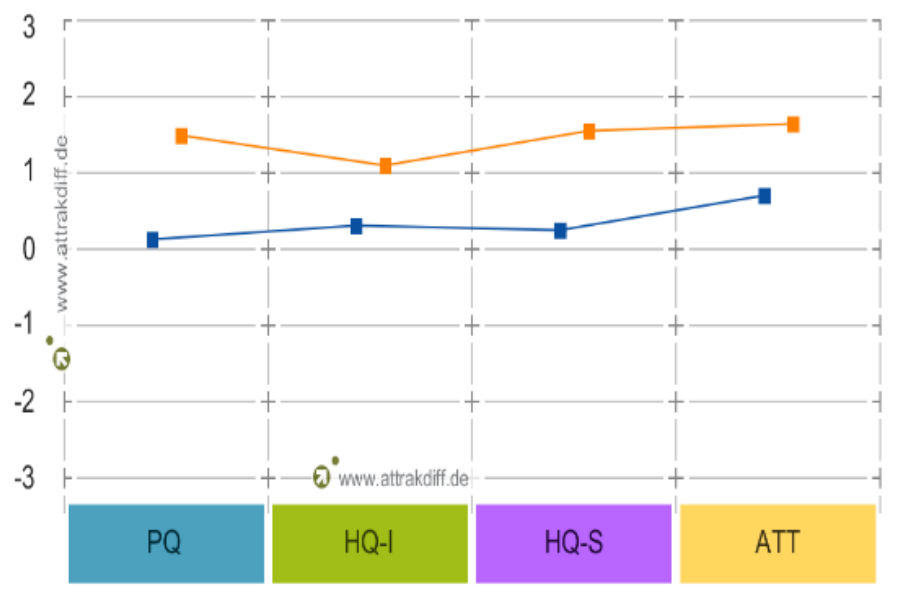

Product:virtual $(n=20)$

PQ:0,15 HQ-1:0,31 HQ-S:0,26 ATT:0,73

--Product:normal $(\mathrm{n}=20)$

PQ:1,51 HQ-l:1,11 HQ-S:1,55 ATT:1,65

[Fig. 3] AttrakDiff Evaluation Results of Virtual YouTuber (VTuber) and General YouTuber

The AttrakDiff evaluation results show that the general YouTuber type is found to have a higher level of satisfaction in all four categories of pragmatic quality (PQ), hedonic quality-identity (HQ-I), hedonic quality-simulation (HQ-S) and attractiveness (ATT) than Virtual YouTuber type. When comparing the categories, the highest difference was found between pragmatic quality (1.36) and hedonic qualitysimulation (1.29). 


\subsubsection{Portfolio Evaluation Results}

As a result of portfolio analysis, it was confirmed that both Virtual YouTuber (VTuber) and General YouTuber had positive results in hedonic quality-identity and hedonic quality-simulation (y-axis) and pragmatic quality (x-axis).

\section{Portfolio-presentation}

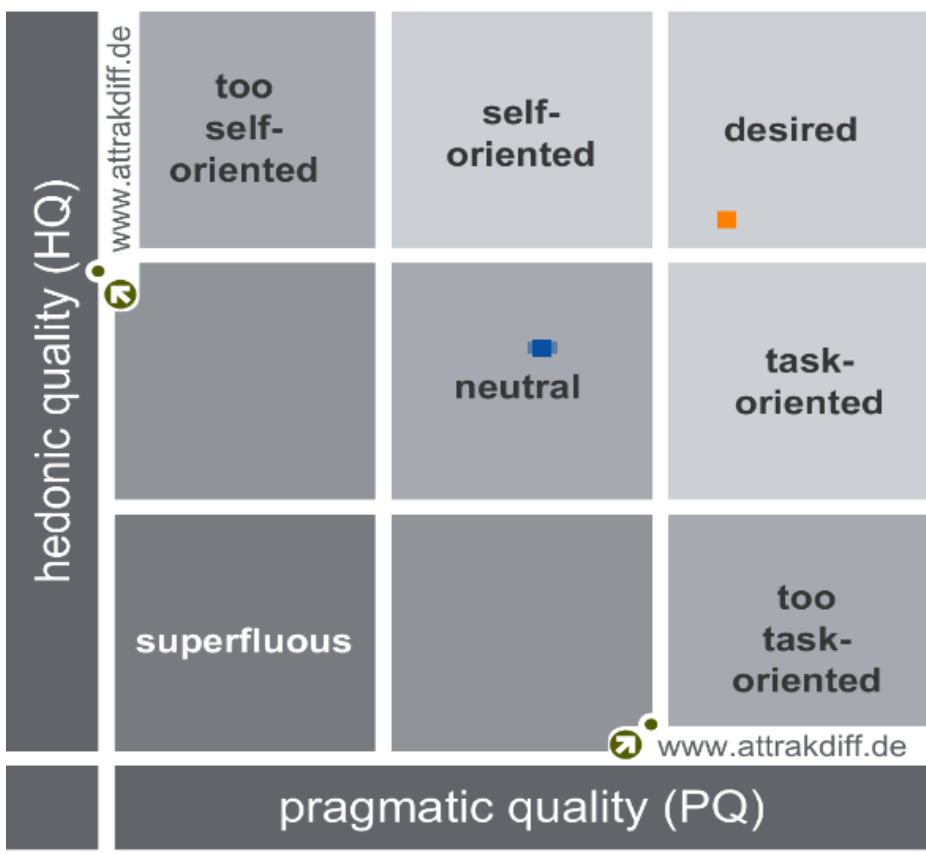

- Product:virtual $(\mathrm{n}=20)$

$P Q: 0,15$ Confidence:0,11

HQ:0,29 Confidence:0,04

- Product:normal $(\mathrm{n}=20)$

$P Q: 1,51$ Confidence:0,04

HQ:1,33 Confidence:0,05

[Fig. 4] Portfolio Evaluation Results of Virtual YouTuber (VTuber) and General YouTuber

Virtual YouTuber (VTuber): The portfolio evaluation result showed that the hedonic quality-identity and hedonic quality-simulation scored 0.29 points with the confidence range of 0.04 , and the pragmatic quality scored 0.15 points with the confidence range of 0.11 . It belonged to the 'neutral' area, as presented in [Fig. 4].

General YouTuber : The portfolio evaluation result revealed that the hedonic quality-identity and hedonic quality-simulation scored 1.33 points with the confidence ranges 0 and 05 , and the pragmatic quality scored 1.51 points with the confidence ranges 0 and 04 . It belongs to the 'ideal' area, as shown in [Fig. 4].

\subsubsection{AttrakDiff Evaluation Results of Virtual YouTuber (VTuber) and General YouTuber (BJ) by Measurement Item}

[Fig. 5] presents the AttrakDiff evaluation results for all 28 pairs of measurement items comparatively between the Virtual YouTuber (VTuber) and the general YouTuber. The VTuber type received a higher 
score than the general YouTuber for the pairs of 'unprofessional-professional' and 'tacky-stylish' in the category of hedonic quality-identity(HQ-I) and 'ugly-attractive' and 'disagreeable-likeable' in the category of attractiveness (ATT).

\section{Description of word - pairs}

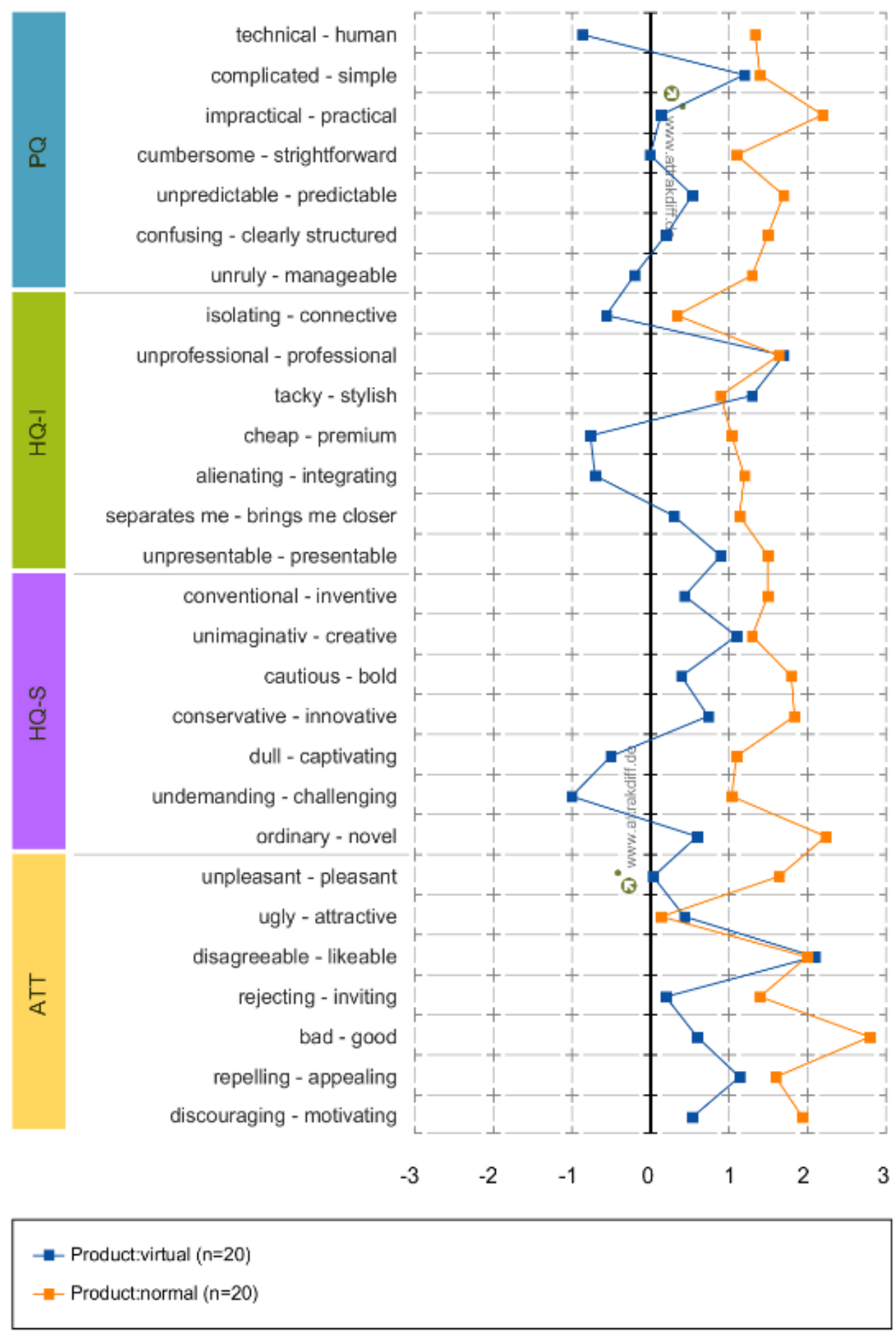

[Fig. 5] AttrakDiff Evaluation Results of Virtual YouTuber (VTuber) and General YouTuber by Measurement Item 


\section{Conclusion}

In this study, the Virtual YouTubers (VTuber) and General YouTuber were compared to show the viewer experience of the content.

This study conducted a survey using AttrakDiff usability evaluation method for ordinary people who have no experience watching virtual YouTubers (VTuber) videos among social media video users. Based on the 50 respondents obtained from the survey, the analysis was conducted by applying an AttrakDiff usability evaluation method that is easy to help users understand how they experience the usability and design of interactive products. The analysis confirmed that the comparison differences between virtual YouTuber (VTuber) and existing general YouTubers were quantitatively and qualitatively, and the evaluation confirmed the characteristics and differences of each of the two groups.

In order to find out the user preferences of virtual YouTuber (VTuber) content, the main conclusions are presented through itemized evaluation, portfolio evaluation, and detailed item evaluation results.

First, the itemized evaluation confirmed that both virtual YouTuber (VTuber) and regular YouTuber were drawing positive responses from viewers. On the other hand, when comparing the means of four items: practicality, identity, irritability, and aesthetics, we found that the positive responses of ordinary YouTubers were significantly higher than those of virtual YouTuber (VTuber).

Second, based on practicality, identity, and stimulation in the portfolio evaluation, we confirmed that virtual YouTubers (VTubers) are located in 'neutral' and general YouTubers are located in 'ideal' areas in portfolio maps. These results seem to make general YouTuber content easier to use than virtual YouTubers (VTubers), and familiar with approaching attractive and forming relationships.

Third, in the detailed item evaluation, the Virtual YouTuber (VTuber) confirmed that the positive responses were higher in the words "professional", "classical" and "appealing" in identity items and the words "appealing" in aesthetic items. These results seem to be attributed to the characteristics of Virtual YouTuber (VTuber) content, which forms virtual characters with motion capture technology and CG technology to host Internet broadcasting.

If we discuss this study, it seems that people in their 20s and 30s in Korea are still more familiar and attracted to ordinary YouTuber than virtual YouTuber (VTuber). In Korea, virtual YouTubers are an unfamiliar field, and since virtual YouTuber (VTuber) started from VR animation, they can vary depending on their experience and preference for animation. Therefore, based on the limitations of this study and these limitations, the following suggestions are made for subsequent studies.

First, this study limited the nationality of the survey subjects to South Korea, but a follow-up study is needed to compare them including those of various nationalities. For example, assuming that Korea and Japan may have different preferences for virtual YouTuber content, it is necessary to find out how the user experience differs when comparing virtual YouTubers and regular YouTubers depending on their cultural characteristics.

Second, this study limited the age group of those surveyed to those in their $20 \mathrm{~s}$ and $30 \mathrm{~s}$, but it would be good to increase the age group from teenagers to those in their $60 \mathrm{~s}$ or older to see if there is a difference between age groups. Since each age group has a different acceptance of technology, there may be differences in the comparison results between virtual YouTuber (Vtuber) and regular YouTubers.

Despite the aforementioned limitations, this study is significant in that it conducted further qualitative studies based on the existing studies which simply compared and analyzed the user response differences between virtual YouTuber (Vtuber) and general YouTubers content with ratings. The results of this study are intended to be used as empirical data for the development of virtual YouTubers. 


\section{References}

[1] J. H. Oh, Realization of a Virtual Celebrity System using Real-time Motion Capture: Focusing on Virtual Youtubers, Soongsil University Graduate School of Information Science, Master's Thesis, (2019)

[2] S. H. Park, E. W. Kang, A Study on the Use of Character Star System to Ease the Entry Barrier of Consumers in Virtual Reality(VR) Content, Journal of the Korean Society of Cartoon \& Animation Studies, (2019), Vol.55, pp.331-356.

[3] Naver, Virtual YouTuber, https://terms.naver.com/entry.nhn?docId=5707663\&cid=43667\&categoryId=43667

[4] J. H. Cho, The influence of the Next-generation VTuber on Contents, Journal of the Korea Institute of Design Research Society, (2019), Vol.4, No.2. pp.173-182. 